\title{
AFLAR and SARAA 2013 Congress - a watershed event for rheumatology in Africa
}

\author{
Girish M. Mody
}

Received: 8 May 2013 / Accepted: 13 May 2013 / Published online: 30 May 2013

(C) Clinical Rheumatology 2013

The combined 7th African League of Associations for Rheumatology (AFLAR) and the 23rd Biennial South African Rheumatism and Arthritis Association congress was held at the Elangeni Hotel in Durban, South Africa from 3 to 6 April 2013. The Elangeni Hotel is situated along the Durban coastline and delegates were able to relax and enjoy the views of the Indian Ocean from their rooms and hear the soothing sounds of the waves along the sea shore-thus enabling them to be refreshed to partake in the scientific programme. The congress also included parallel sessions for Paediatric Rheumatology and Health Professionals in Rheumatology.

The inaugural meeting of AFLAR was held in 1989; thus, the 2013 congress marked the 25th anniversary of the formation of AFLAR. The first AFLAR congress was held in Egypt in 1991 and the subsequent congresses were held every 4 years in Tunis, Tunisia (1995); Cape Town, South Africa (1999); Rabat, Morocco (2003); Nairobi, Kenya (2007) and Algiers, Algeria (2011). In 2007, a decision was taken at the congress in Nairobi, Kenya that after the 2011 Congress in Algeria, the AFLAR congresses would be held biennially and that the term of the president and executive committee of AFLAR would be for 2 years instead of the previous 4-year term of office. I have had the privilege of attending all the congresses of AFLAR since 1991 and also serving as president-elect from 1995 to 1999 and then as president from 1999 to 2003. During the 25 years since the formation of AFLAR, the major health challenges for Africa have included the HIV epidemic, tuberculosis, malaria and malnutrition. However, over the past decade, and especially over the past 5 years, there has been a considerable growth in interest in rheumatology from many regions in Africa. On this occasion, I had the challenge and

G. M. Mody $(\bowtie)$

School of Clinical Medicine, College of Health Sciences, University of KwaZulu-Natal, Durban, South Africa

e-mail: modyg@ukzn.ac.za honour of chairing the organising committee for the 2013 congress which included Doctors Neeta Patel, Thozama Dubula, Rasha Maharaj, Amritha Budhoo, Keith Chinniah, Kogie Chinniah and Mohamed Akhalwaya, and $\mathrm{Sr}$ Nokujabula Mkhize.

Currently, regular rheumatology congresses are held in countries such as Egypt, Tunisia, Morocco, Libya, Algeria, Kenya and South Africa, either annually or biennially. In addition, rheumatology educational meetings were held in Khartoum, Sudan and Lagos, Nigeria during 2009 and in Lusaka, Zambia in 2010. Despite the growing interest in pursuing a career in rheumatology and the tremendous advances which have been made in recent years, there are still many countries in sub-Saharan Africa which have either no rheumatologists or a very few rheumatologists to manage the burden of rheumatic diseases. Moreover, in most countries, there are limited resources for basic autoantibody tests, imaging such as ultrasound or magnetic resonance imaging, disease modifying drug therapy and access to arthroplasty.

There are also many factors that make it very difficult for doctors in Africa to participate in regional meetings. Firstly, for many of the countries, travel within Africa is very costly and is often more expensive than travel to countries in Europe. Secondly, there are two major languages used in academic meetings - namely English and French. Thus, unless simultaneous translation facilities are available, language can also be a deterrent to participate in meetings within Africa. Thirdly, the support from pharmaceutical industry to participate in congresses is considerably less, or in some cases nonexistent, as many of the companies do not have subsidiaries in the African countries as they do not provide a market for their products.

The organising committee was fortunate to obtain support from some of the pharmaceutical companies which made it possible to provide English-French translation throughout the plenary sessions of the congress, provide partial fellowships for over 25 delegates from all over Africa and also host a 
distinguished international faculty for the congress. In addition, the 3-day scientific programme provided an opportunity for delegates to present their own research work as oral presentations or posters.

One of the major successes of the congress was the presence of delegates from a large number of countries within Africa, especially sub-Saharan Africa. We were able to attract delegates from Algeria, Benin, Burkina-Faso, Cameroon, Congo Brazzaville, Democratic Republic of Congo (DRC), Kenya, Madagascar, Mali, Mauritius, Namibia, Nigeria, Tanzania, Zambia, Zimbabwe and South Africa. There were more than 250 participants in the congress with 15 international contributors from nine different countries and delegates from nearly 20 countries.

It was also very encouraging to receive a large number of abstracts for the congress and 59 were accepted as free paper presentations and 46 as posters. As we are in a developmental era in the African setting, we had a larger number of abstracts related to infections. As new rheumatology clinics and services are being developed in many countries, delegates reported audits of their clinics and described the spectrum of musculoskeletal disorders in local communities and presented the results of surveys to determine the prevalence, spectrum and burden of musculoskeletal symptoms in countries where such services and information did not exist. In addition there were also studies relating to epidemiology, genetics, evaluation of measures to assess disease activity, use of imaging such as ultrasound, treatment and outcome.

The organising committee is also grateful to Professor Maxime Dougados, President of the European League Against Rheumatism (EULAR), and the EULAR executive committee, who agreed to sponsor a symposium during the congress. The EULAR symposium was addressed by Professors Maurizio Cutolo (Pathophysiological and clinical basis for the EULAR recommendations in rheumatoid arthritis), Hans Bijlsma (What can EULAR offer you in Education?) and Daniel Aletaha (The principles and methods of deriving the EULAR recommendations). They also contributed to the scientific programme with their excellent presentations on "Treatment of osteoarthritis" (JWJ Bijlsma), "From Raynaud's to systemic sclerosis: the diagnostic / prognostic role of capillaroscopy" (M Cutolo) and "Treatment of rheumatoid arthritis: clinical, ethical and economic challenges" (D Aletaha).

Members of the ILAR-East Africa Initiative from North America have been passionate about promoting rheumatology and contributing to rheumatology education and training in Africa. They volunteered to contribute to the academic programme and endeared themselves to many of their delegates by their participation during the entire academic programme, their interaction with the delegates and their desire and commitment to assist with the training of rheumatologists in Africa.
Professor Dan Sager from Oregon, USA, discussed a simplified approach to the differential diagnosis of rheumatic diseases during his address on "A methodology for rheumatologic diagnosis at point of care". He also presented an update on "Mobile technologies for clinical support at point of care". Professor Henri Menard, from McGill University, emphasised the importance of clinical assessment in patients with low back pain during his presentation on "An Oslerian approach to low back pain" and discussed the role anti-Sa antibodies during his eloquent address on "Auto-antibodies in rheumatoid arthritis (RA): A roadmap to personalized medicine". Professor Ines Colmegna, also from McGill University, provided an update on the effects of ageing on the immune system and reviewed the mechanisms of action and effects of methotrexate. She highlighted the current research agenda to address many unanswered questions relating to methotrexate. Professor Alessandra Bruns, from Sherbrooke University in Canada, addressed the paediatric rheumatologists on the important role of ultrasound in the evaluation of children and also conducted a practical workshop on the use of ultrasound in children. She discussed the role of ultrasound in crystal arthropathies during the plenary session of the congress.

Professors Ernest Choy (UK), Rieke Alten (Germany) and Flip Van Den Bosch (Belgium) participated in industrysponsored symposia and also addressed the subjects of fibromyalgia, osteoporosis and comprehensive management of RA, respectively, during the scientific programme. Professor David D'Cruz, Clinical Team Lead at the internationally renowned Lupus Unit at St Thomas's Hospital in London, spoke on "Systemic lupus erythematosus (SLE) - An update" and "Vasculitis".

Professors Helen Foster (UK) and Ricardo Russo (Argentina) contributed to the paediatric rheumatology sessions during the congress. Professor Foster addressed the conference on "Juvenile Idiopathic Arthritis (JIA)Update", "Transitional care in adolescents" and "Access to care, education, teaching, training and collaboration in paediatric rheumatology". Professor Russo discussed "Auto-inflammatory disorders in children".

Mr Benjamin Smith, past president of the Association of Rheumatology Health Professionals in the USA, discussed "The role of Physician Assistants and Nurse Practitioners in Rheumatology Practice" during the plenary session of the congress. He also addressed the health professionals on "Why do my joints hurt? Using case studies to Review Osteoarthritis (OA) and RA Diagnostic and Treatment Guidelines" and "Training and Using Physician Assistants and Nurse Practitioners effectively in a Rheumatology Practice". Sr Elizabeth McIvor from Glasgow has a long record of contribution to health professional education and training in Africa. She provided an update on the status of "Rheumatology Nursing in the UK" and also discussed the "Assessment of Outcomes in Rheumatology". 
Divengi-Nzambi et al. reported a large epidemiological survey of an urban and rural population of 6,500 people in the DRC and found that the prevalence of RA was $0.9 \%$ in Kinshasa and $1.4 \%$ in the rural area of Nkamba, in contrast to earlier studies which noted that RA was rare in rural communities (FP21). The prevalence and spectrum of musculoskeletal disorders in the rural sample of 1,500 people of the DRC showed that $48.9 \%$ had a rheumatic complaint, and that the commonest disorder was osteoarthritis, which was present in $36.8 \%$ of the population. A prospective study of 3,384 participants in Nairobi by Ekwom et al. (FP3) found that musculoskeletal symptoms were present in $12 \%$ of the population. Trollip et al. observed a change in the profile of rheumatic diseases in Zambia where HIVassociated spondyloarthritis accounted for nearly $50 \%$ of the patients in the 1990s (FP4). Over the past 3 years, the commonest diseases seen in the same referral centre were $\mathrm{RA}, \mathrm{OA}$ and connective tissue diseases.

Doulla et al. (FP1) studied a group of 62 patients with HCV infection in Cameroon and noted that musculoskeletal manifestations were present in $24.2 \%$ with arthralgia, arthritis and fatigue being the most common manifestations at presentation. Okwara et al. from Nigeria found that articular disorders were commoner in a series of $240 \mathrm{HIV}$-positive patients compared to controls (FP33). Njobvu et al. reported that spondyloarthritis $(\mathrm{SpA})$ and muscle diseases were the commonest rheumatic diseases in HIV-infected patients in Zambia and that drug toxicity, especially zidovudine myopathy, was not uncommon. The impact of HIV infection in patients with RA and SLE were presented by Patel et al. (FP35) and Ntumba et al. (FP 36) in Durban and Johannesburg, South Africa, respectively.

In a series of 128 patients with RA in Kinshasa, DRC, Malemba et al. (FP2) found that $<50 \%$ of their patients had a positive rheumatoid factor or anti-CCP. They also noted that severe joint damage was uncommon, despite a delay in presentation, and the HLA-DR $\beta 1$-shared epitope was uncommon, suggesting that their patients had a different genotype. Govind et al. showed differences in the susceptibility variants of the HLA class II genes between Europeans and Black South Africans and also found that the presence of valine at position 11 of the HLA DR $\beta 1$ molecule was associated with the strongest risk for RA whereas serine confers protection (FP6, FP18).

Hodkinson et al. showed that in a low resource setting in Johannesburg, South Africa, the clinical disease activity index (CDAI) was an acceptable alternative to simplified disease activity index (SDAI) in measuring disease activity, and that a tight control strategy could achieve short-term remission of $29 \%$ in the absence of biological agents (FP38). Slimani et al. also found a strong correlation between SDAI and CDAI, and DAS28, in their multicenter study in Algeria, and they proposed higher cutoff values to ensure appropriate categorization of their patients. In the same study, they also found that patients with lower formal education were likely to have a poorer outcome due to more active and more severe disease (P36).

The profiles of patients with RA and the pivotal role of methotrexate were reported in series from Zambia (FP23), Cameroon (FP39), Nigeria (P20) and the DRC (FP40 and P21). In the DRC (FP40), patients showed a response to lower weekly doses of methotrexate (average of $9.7 \mathrm{mg}$ after 20 months). A study on the cardiovascular risk factors in RA in Kenya noted that there was a significant increase in the prevalence of hypertension in $41.3 \%$ of their patients compared to controls.

A review of 167 hospitalised SLE patients in Durban, South Africa by Dubula et al. found that $35.9 \%$ of the hospital admissions were for infections and the most common sites were respiratory, soft tissue and the urinary tract (FP28). Tuberculosis was diagnosed in $16.7 \%$ of all hospital admissions. Karolia et al. reported a prospective study of 57 hospitalised SLE patients and also found that active disease and infections were the commonest reasons for admission (FP31). Budhoo et al. showed that there was no difference in the severity of SLE among Indians and Black Africans in Durban, South Africa, and apart from an increase in oral ulcers and thrombocytopaenia in Indians, and serositis and anti-Sm antibodies in Black Africans, there were no other differences. Mody et al. analysed their series of 124 patients with lupus nephritis in Durban and found that membranous nephritis was significantly more common when compared to data in Caucasians, and that mycophenolate mofetil was effective as induction and maintenance therapy (FP29). Frost et al. noted that FC gamma receptor IIB rs1050501 polymorphisms are a possible risk factor for SLE in Blacks South Africans (P5). The spectrum of manifestations of SLE was reported from Cameroon (P1), Cape Town, South Africa (P4), Nigeria (P7) and Zambia (P3).

The overall spectrum of rheumatic diseases seen in hospital-based populations in Mali (FP7), Kenya (P35) and the DRC (P33) and the spectrum of connective tissue disease seen in rheumatology clinics in Cape Town (P12), Lusaka (P13) and Nigeria (P14) were also presented.

Lumbamba et al. found that the prevalence of the metabolic syndrome was $51 \%$ in their series of 200 patients with gout at the Tygerberg Hospital in Cape Town (FP37). A prospective survey of osteoporotic hip fractures by Paruk et al. in Durban, South Africa showed that there was a high mortality (34.6\% at 12 months) and high morbidity (FP13).

Slimani et al. studied 192 knees of 102 patients with OA in Algeria and showed that osteophytes were detected 2.5 times more often with musculoskeletal ultrasound (FP16). In another Algerian study, the calculation of a simplified enthesitis ultrasound score was found to be a sensitive and specific measure for the diagnosis of a SpA (FP17). Benitha 
et al. analysed the time and cost associated with ultrasound training and the impact of the use of ultrasound on clinical decision making (FP15).

Some of the findings relating to lumbar spinal disorders were that chronic low back pain was associated with a neuropathic component in $28 \%$ of 167 patients in Cameroon (FP9), the efficacy of facet joint injections in providing symptomatic relief in Benin (FP11), the spinal canal dimensions may be lower in Congolese patients in the DRC (FP10) and that the L4-L5 level was found to be the commonest level for disc herniation in Brazzaville, Congo (FP 12). The risk factors, functional evaluation, clinical and radiological features of knee osteoarthritis were studied in Congo Brazzaville (FP32), DRC (FP25 and FP41) and Nigeria (FP42).

Some of the interesting observations in the poster presentations were the occurrence of OA of the interphalangeal joints of the thumb related to repetitive manual activities (P24), a case series of eight patients with the increasingly recognised posterior reversible encephalopathy syndrome in SLE (P6), the occurrence of macrovascular disease in eight patients with scleroderma (P16) and osteoarticular sarcoidosis in four patients with HIV infection (P28).

This congress also marked the first gathering of paediatric rheumatologists from Kenya, Zambia, Tanzania and South Africa at a meeting of AFLAR. The spectrum of rheumatological conditions seen in hospital-based studies of children was reported from Cameroon (FP44, FP49), Kenya (FP48) and Zambia (FP43). Singwe-Ngandeu et al. noted that the commonest conditions seen in children in Yaounde, Cameroon were related to infection in $45 \%$ followed by JIA in $16 \%$ (FP49) while Doulla et al. noted that the commonest conditions seen in Doula, Cameroon were mechanical lumbar pain, chronic inflammatory arthri- tis and peripheral joint disorders (FP44). Chipeta et al. analysed the findings of 126 children with chronic arthritis in Zambia. They found that $16.3 \%$ were HIV positive, eye involvement occurred in $17.6 \%$ and the commonest types of joint involvement were polyarticular rheumatoid factornegative JIA and oligoarthritis. Mwaba et al. studied 146 children with acute pharyngitis and noted that $15.1 \%$ had group A beta-hemolytic streptococcus which was sensitive to penicillin (FP53). Scott et al. presented a series of 15 patients with HIV-associated infection in Cape Town, South Africa (FP54).

During the parallel SAHPR session, the assessment of compliance (FP55) and the socio-demographic characteristics and co-morbidities (FP59) of a series of 54 patients from the rheumatology clinic in Cape Town were presented. Other presentations included the use of an accelerometer for measuring habitual physical activity in RA (FP57) and assessment of the physical, biomechanical and mood profile of patients with ankylosing spondylitis (FP58).

One of the social highlights of the congress was a cocktail and dinner evening at the iconic Moses Mabhida Stadium in Durban, one of the venues for the 2010 FIFA Soccer World Cup. All the delegates had an opportunity to follow in the path of Ronaldo, Messi and other soccer legends and walk through the players' tunnel to the edge of the field, sip a cocktail and enjoy the magnificent views of the overhead arch, a privilege usually reserved for players, officials and dignitaries.

The 2015 AFLAR congress will be held in Nigeria and I hope that the momentum associated with the success of the 2013 congress propels AFLAR to greater heights.

Disclosures Member of the VIMOVO advisory board for AstraZeneca. 